\title{
Higher order perturbations of anti-de Sitter space and time-periodic solutions of vacuum Einstein equations
}

\author{
Andrzej Rostworowski* \\ M. Smoluchowski Institute of Physics, Jagiellonian University, 30-348 Kraków, Poland \\ and Theoretical Physics Department, CERN, CH-1211 Geneva 23, Switzerland
}

(Received 31 March 2017; published 26 June 2017)

\begin{abstract}
Motivated by the problem of stability of anti-de Sitter (AdS) spacetime, we discuss nonlinear gravitational perturbations of maximally symmetric solutions of vacuum Einstein equations in general and the case of AdS in particular. We present the evidence that, similarly to the self-gravitating scalar field at spherical symmetry, the negative cosmological constant allows for the existence of globally regular asymptotically AdS, time-periodic solutions of vacuum Einstein equations whose frequencies bifurcate from linear eigenfrequencies of AdS. Interestingly, our preliminary results indicate that the number of oneparameter families of time-periodic solutions bifurcating from a given eigenfrequency equals the multiplicity of this eigenfrequency.
\end{abstract}

DOI: $10.1103 /$ PhysRevD.95.124043

\section{INTRODUCTION}

The problem of late-time dynamics in asymptotically anti-de Sitter (AdS) spacetimes received quite a lot of attention in the past five years and revealed a few surprises. First, paper [1] provided numerical and heuristic evidence for two types of possible scenarios in the model EinsteinAdS-massless scalar field system at spherical symmetry: (1) turbulent dynamics leading to concentration of (a fraction of) energy on small spatial scales leading to a black hole formation on the time scale $\mathcal{O}\left(\epsilon^{-2}\right)$, where $\epsilon$ measures the amplitude of initial data and (2) quasiperiodic evolution. Second, stable time-periodic solutions for this model were discovered [2], providing an explanation for quasiperiodic evolution, as being anchored to stability islands of these time-periodic solutions (see also [3] vs [4]) and an important role of the nondispersive spectrum of linear perturbations for instability was identified [5,6]. Finally, a new perturbation scheme was proposed [7] and firmly developed [8,9] to capture the dynamics on the $\mathcal{O}\left(\epsilon^{-2}\right)$ time scale. It consists in setting a time-averaged system of equations, for the slow time dependence of Fourier-like coefficients ("slow" with respect to the "fast" time scale of Fourier modes themselves). The main advantage of this system (hereafter referred to as the resonant system) is that it has a scaling symmetry: if its solution with the initial amplitude 1 does something at time $t$, then the corresponding solution with the initial amplitude $\epsilon$ does the same thing at time $t / \epsilon^{2}$. Thus, by solving the

*arostwor@th.if.uj.edu.pl

Published by the American Physical Society under the terms of the Creative Commons Attribution 4.0 International license. Further distribution of this work must maintain attribution to the author(s) and the published article's title, journal citation, and DOI. resonant system one can probe the regime of arbitrarily small perturbations (whose outcome of evolution of full Einstein equations is beyond the possibility of numerical verification). Interestingly, the solutions of the resonant system generically develop an oscillatory blow-up in finite time giving an extra piece of evidence for instability of AdS [10].

After gaining some insights on the AdS stability problem from a toy model of a matter field coupled to Einstein equations at spherical symmetry, it becomes crucial to decide if it constitutes a good model for a general setting and for Einstein equations in vacuum in particular. Extrapolating from $[1,2]$ beyond spherical symmetry, one could make the following two conjectures (for effectively reflecting boundary conditions at timelike boundary of AdS): (1) the turbulent instability is present in AdS, i.e., the transfer of energy to arbitrarily high frequencies does not saturate and probably leads to a gravitational collapse, (2) there exist globally regular, time-periodic (TP), asymptotically AdS (aAdS) solutions of Einstein equations (TP solutions, also refereed to as geons in [11-13]), immune to this instability; their frequencies are expected to bifurcate from eigenfrequencies of the spectrum of linear perturbations of AdS. One possible way to build evidence for these conjectures is to set the resonant system for vacuum Einstein equations (to study instability) and to construct TP solutions perturbatively. To achieve these goals one has to master gravitational perturbations of $\mathrm{AdS}$ (in principle, up to arbitrarily high orders to construct TP solutions, but only up to the third order to construct the resonant system).

There is a limited number of results on the AdS instability outside spherical symmetry. Horowitz and Santos showed numerically that in the vacuum case there are linear modes that can be extended to form TP solutions [11]. The results of perturbative calculations performed around some single modes (or some linear combination of 
modes) up to the third order were given in [12] and its sequel [13]. However a systematic approach to gravitational perturbations of AdS has never been presented. The main aim of this work is to fill in this gap and to treat higher order perturbations of AdS in a systematic manner. We hope that our results will provide a solid base for the future construction of the resonant system for vacuum AdS and for more systematic studies of regular, asymptotically AdS, time-periodic solutions of Einstein vacuum equations. We restrict ourselves to the case of axially symmetric perturbations as a relatively simple and illustrative example. All conceptual difficulties are encountered when stepping out from spherical symmetry to axial symmetry. Once this model case is well understood, adding azimuthal angle dependence (in $3+1$ dimensions) is a technical, not a conceptual, issue.

The novel feature of vacuum perturbation with respect to the spherically symmetric self-gravitating scalar field model is the degeneracy in the spectrum of linear perturbations. Namely, the spectrum of linear perturbation in $3+1$ dimensions reads $\omega_{\ell, j}=q+\ell+2 j$, where $\ell$ is the mode angular momentum index, $j$ is the nodal number of the corresponding radial wave function, and $q$ reads 1 or 2 for polar and axial modes, respectively. It was noted with a bit of surprise that only in special cases (listed in Sec. VI in [13]) linear eigenmodes do admit a nonlinear extension to a TP solution. However, it is well known since quantum mechanics that the degeneracy case may require a special treatment in the perturbation expansion, and a more appropriate question to ask is which linear frequencies (rather then which linear modes) are bifurcation points for TP solutions. It seems that, similarly to the spherically symmetric scalar field case, there are one-parameter families of TP solutions, bifurcating from each linear frequency and the number of these families of TP solutions is equal to the multiplicity of the linear eigenfrequency [14]. In fact, the key property allowing the time-periodic solutions of the type studied in [2] (i.e., bifurcating from a single linear eigenfrequency) to exist, is the absence of $(+++)$ resonances; see [8] for the definition and a brute force proof for the Einstein-AdSmassless scalar field system (for a deeper discussion and the proof of this property for a test scalar field on AdS background see [15] and references therein). It seems that there are no $(+++)$ resonances also in the vacuum AdS case and, in this sense, the self-gravitating scalar field seems to be a good toy model to study the gravitational stability of AdS.

We concentrate in this work on (nonlinear) perturbations of AdS space; however, it is quite clear that a similar approach can be taken for perturbations of any spherically symmetric solution of vacuum Einstein equations. In fact all formulas in the paper, that are not specific to a particular asymptotic structure of the spacetime, are general enough to work for any maximally symmetric solution of vacuum
Einstein equations (anti-de Sitter, Minkowski and de Sitter) in static coordinates, i.e., with the line element in the form (10). The key observation is to split perturbative Einstein equations into these satisfied identically by a suitable choice of master scalar variables (see below) and those that govern the dynamics of these master scalar variables. To the best of our knowledge this splitting has never been highlighted in the literature.

The paper is organized as follows. In Sec. II we discuss the general setup for perturbation expansion around exact solutions of vacuum Einstein equations and ReggeWheeler [16] decomposition of metric perturbations. In Secs. III and IV we discuss polar- and axial-type perturbations of maximally symmetric spacetimes. We apply the formalism of these sections in Sec. V to construct and study some properties of globally regular, aAdS, time-periodic solutions of vacuum Einstein equations. Then we conclude in Sec. VI.

\section{AdS $_{d+1}$ PERTURBATION IN VACUUM: GENERAL SETUP}

We are interested in solutions of vacuum Einstein equations with negative cosmological constant $\Lambda=-\frac{d(d-1)}{2 \mathfrak{l}^{2}}$ :

$$
R_{\mu \nu}+\frac{d}{\mathfrak{l}^{2}} g_{\mu \nu}=0 .
$$

Let the "bar" quantities stand for the AdS quantities (i.e., $\bar{g}_{\mu \nu}, \bar{g}^{\mu \nu}, \bar{\Gamma}_{\mu \nu}^{\alpha}, \bar{R}_{\mu \nu}$ are AdS quantities). Now let $g_{\mu \nu}=$ $\bar{g}_{\mu \nu}+\delta g_{\mu \nu}$ or in matrix notation $g=\bar{g}+\delta g$, where $\delta g_{\mu \nu}$ will be expanded as

$$
\delta g_{\mu \nu}=\sum_{1 \leq i}^{(i)} h_{\mu \nu} \epsilon^{i}
$$

later on. Then we have

$$
\begin{aligned}
g^{\alpha \beta}= & \left(\bar{g}^{-1}-\bar{g}^{-1} \delta g \bar{g}^{-1}+\bar{g}^{-1} \delta g \bar{g}^{-1} \delta g \bar{g}^{-1}-\ldots\right)^{\alpha \beta} \\
= & \bar{g}^{\alpha \beta}+\delta g^{\alpha \beta} \\
\Gamma_{\mu \nu}^{\alpha}= & \bar{\Gamma}_{\mu \nu}^{\alpha}+\frac{1}{2}\left(\bar{g}^{-1}-\bar{g}^{-1} \delta g \bar{g}^{-1}+\bar{g}^{-1} \delta g \bar{g}^{-1} \delta g \bar{g}^{-1}-\ldots\right)^{\alpha \lambda} \\
& \times\left(\bar{\nabla}_{\mu} \delta g_{\lambda \nu}+\bar{\nabla}_{\nu} \delta g_{\lambda \mu}-\bar{\nabla}_{\lambda} \delta g_{\mu \nu}\right) \\
= & \bar{\Gamma}_{\mu \nu}^{\alpha}+\delta \Gamma_{\mu \nu}^{\alpha} \\
R_{\mu \nu}= & \bar{R}_{\mu \nu}+\bar{\nabla}_{\alpha} \delta \Gamma_{\mu \nu}^{\alpha}-\bar{\nabla}_{\nu} \delta \Gamma_{\alpha \mu}^{\alpha}+\delta \Gamma_{\alpha \lambda}^{\alpha} \delta \Gamma_{\mu \nu}^{\lambda}-\delta \Gamma_{\mu \alpha}^{\lambda} \delta \Gamma_{\lambda \nu}^{\alpha} \\
= & \bar{R}_{\mu \nu}+\delta R_{\mu \nu} .
\end{aligned}
$$

Equation (3) is straightforwardly obtained by recursive application of the formula $g^{\alpha \beta}=\bar{g}^{\alpha \beta}-\bar{g}^{\alpha \mu} \delta g_{\mu \nu} g^{\nu \beta}$ and then Eqs. (4) and (5) easily follow. Thus, the Einstein equations read (bar quantities are solutions to Einstein equations) 


$$
\delta R_{\mu \nu}+\frac{d}{\mathfrak{l}^{2}} \delta g_{\mu \nu}=0 .
$$

Now, plugging the expansion (2) into (6) and collecting the terms at the same powers of $\epsilon$, we get the following hierarchy of equations:

$$
{ }^{(i)} E_{\mu \nu}:=\Delta_{L}{ }^{(i)} h_{\mu \nu}-{ }^{(i)} S_{\mu \nu}=0 .
$$

The Lorentzian Lichnerowicz operator $\Delta_{L}$ reads

$$
\begin{aligned}
\Delta_{L} h_{\mu \nu} & =\frac{1}{2} \bar{g}^{\alpha \lambda}\left(\bar{\nabla}_{\alpha}\left(\bar{\nabla}_{\mu} h_{\lambda \nu}+\bar{\nabla}_{\nu} h_{\lambda \mu}-\bar{\nabla}_{\lambda} h_{\mu \nu}\right)-\bar{\nabla}_{\nu}\left(\bar{\nabla}_{\alpha} h_{\lambda \mu}+\bar{\nabla}_{\mu} h_{\lambda \alpha}-\bar{\nabla}_{\lambda} h_{\alpha \mu}\right)\right)+\frac{d}{\mathfrak{l}^{2}} h_{\mu \nu} \\
& =\frac{1}{2}\left(-\bar{\nabla}^{\alpha} \bar{\nabla}_{\alpha} h_{\mu \nu}-\bar{\nabla}_{\mu} \bar{\nabla}_{\nu} h-2 \bar{R}_{\mu \alpha \nu \beta} h^{\alpha \beta}+\bar{\nabla}_{\mu} \bar{\nabla}^{\alpha} h_{\nu \alpha}+\bar{\nabla}_{\nu} \bar{\nabla}^{\alpha} h_{\mu \alpha}\right),
\end{aligned}
$$

where $h=\bar{g}^{\alpha \beta} h_{\alpha \beta}, h^{\alpha \beta}=\bar{g}^{\alpha \mu} \bar{g}^{\beta \nu} h_{\mu \nu}$ and we have used

$$
\begin{aligned}
\left(\bar{\nabla}_{\alpha} \bar{\nabla}_{\nu}-\bar{\nabla}_{\nu} \bar{\nabla}_{\alpha}\right) h_{\mu}^{\alpha} & =\bar{R}_{\beta \alpha \nu}^{\alpha} h_{\mu}^{\beta}-\bar{R}_{\beta \mu \alpha \nu} h^{\alpha \beta} \\
& =-\frac{d}{\mathfrak{l}^{2}} h_{\nu \mu}-\bar{R}_{\beta \mu \alpha \nu} h^{\alpha \beta}
\end{aligned}
$$

The source terms in (7) read

$$
\begin{aligned}
{ }^{(i)} S_{\mu \nu}= & {\left[\epsilon^{i}\right]\left\{-(1 / 2) \bar{\nabla}_{\alpha}\left[\left(-\bar{g}^{-1} \delta g \bar{g}^{-1}+\bar{g}^{-1} \delta g \bar{g}^{-1} \delta g \bar{g}^{-1}-\cdots\right)^{\alpha \lambda}\left(\bar{\nabla}_{\mu} \delta g_{\lambda \nu}+\bar{\nabla}_{\nu} \delta g_{\lambda \mu}-\bar{\nabla}_{\lambda} \delta g_{\mu \nu}\right)\right]\right.} \\
& +(1 / 2) \bar{\nabla}_{\nu}\left[\left(-\bar{g}^{-1} \delta g \bar{g}^{-1}+\bar{g}^{-1} \delta g \bar{g}^{-1} \delta g \bar{g}^{-1}-\cdots\right)^{\alpha \lambda}\left(\bar{\nabla}_{\mu} \delta g_{\lambda \alpha}+\bar{\nabla}_{\alpha} \delta g_{\lambda \mu}-\bar{\nabla}_{\lambda} \delta g_{\mu \alpha}\right)\right] \\
& \left.-\delta \Gamma_{\alpha \lambda}^{\alpha} \delta \Gamma_{\mu \nu}^{\lambda}+\delta \Gamma_{\mu \alpha}^{\lambda} \delta \Gamma_{\lambda \nu}^{\alpha}\right\}
\end{aligned}
$$

where $\left[\epsilon^{i}\right] f$ denotes the coefficient at $\epsilon^{i}$ in the (formal) power series expansion of $f=\sum_{i} f_{i} \epsilon^{i}$. The formulas (7)(9) are in fact completely general, i.e., they work for any value of the cosmological constant and any zero order solution, no matter if spherically symmetric or not. From now on we limit to $d=3$ spatial dimensions.

Before presenting all further technicalities let us start with discussing general strategy. We will follow the Regge-Wheeler (RW) seminal paper [16] and use spherical symmetry of the zero order solution (AdS in our case) to expand the metric perturbations into scalar, vector, and tensor spherical harmonics. The reader unfamiliar with the RW decomposition can consult Sec. II of the excellent review by Nollert [17]. After separating angular dependence, the system of 10 perturbative Einstein equations (7) splits into the system of seven equations for polar (alternatively called scalar) type perturbations and the system of three equations for axial (alternatively called vector) type perturbations $[16,17]$. The polar and axial parts decouple at linear order, but generally mix at higher orders. The axial symmetry is exceptional in this respect, as if we excite only polar perturbations at linear order we stay in the polar sector at all higher orders as well, while starting with only axial perturbations at linear order results in axial perturbations at all odd orders and polar perturbations at all even orders of perturbation expansion.
The most general gauge vector (for given spherical harmonics indexes $\ell, m$ ) splits accordingly into polar and axial parts parametrized by three and one function, respectively. It turns out that the Lichnerowicz operator (8) depends only on four and two RW gauge invariant variables in polar and axial sectors, respectively, while the sources (9) contain also gauge degrees of freedom. After introducing RW variables the system is still messy; however, it is well known that at linear order both polar and axial sector are governed by only one (for given $\ell, m$ ) master scalar variable each. It is very convenient to introduce corresponding scalar variables also at higher orders-not only to ease the solution of the system of Einstein equations but also to make the resonant structure of these equations explicit. There are, however, a few problems to be faced here:

1. to find the correct definition of master scalar variables at higher orders,

2. to build the source term for the (inhomogeneous) wave equation for this scalar variable from the sources (9) in perturbative Einstein equations (7),

3. to be able to switch between the components of metric perturbations and the master scalar variables (to solve Einstein equations easily) and back (to reconstruct the metric perturbations from scalar variables and to be able to find the sources (9) necessary 
to step to the next order of the perturbation expansion),

4. to set the metric perturbations to the aAdS form with a suitable gauge transformation (it is necessary to insure the correct asymptotics of the sources to step to the next order),

5. to treat the special cases $\ell=0$ (for polar-type perturbations only) and $\ell=1$ (both for polar- and axial-type perturbations); they correspond to gauge degrees of freedom at linear order but have to be correctly dealt with at higher orders.

In the two following sections we discuss solutions to all problems listed above for axially symmetric perturbations starting from polar- and axial-type perturbations at linear order. In what follows we use coordinates $(t, r, \theta, \phi)$ in which the AdS line element reads

$$
d s^{2}=-A d t^{2}+A^{-1} d r^{2}+r^{2} d \Omega_{2}^{2},
$$

with $A \equiv A(r)=\left(1+r^{2} / \mathfrak{l}^{2}\right)$. Then we use Mukohyama [18], Appendix B's definitions for scalar, vector, and tensor spherical harmonics (up to normalization factors). In $3+1$ dimensions any tensor $T_{\mu \nu}$ can be split into seven polar and three axial components. For any tensor $T_{\mu \nu}$, its polar components expanded into (one scalar-, one vector-, and two tensor-types) polar spherical harmonics at axial symmetry read

$$
\begin{aligned}
T_{a b}(t, r, \theta) & =\sum_{\ell} T_{\ell a b}(t, r) P_{\ell}(\cos \theta), \quad a, b=0,1, \\
T_{a 2}(t, r, \theta) & =\sum_{\ell} T_{\ell a 2}(t, r) \partial_{\theta} P_{\ell}(\cos \theta), \quad a=0,1, \\
\frac{1}{2}\left(T_{22}(t, r, \theta)+\frac{T_{33}(t, r, \theta)}{\sin ^{2} \theta}\right) & =\sum_{\ell} T_{\ell+}(t, r) P_{\ell}(\cos \theta), \\
\frac{1}{2}\left(T_{22}(t, r, \theta)-\frac{T_{33}(t, r, \theta)}{\sin ^{2} \theta}\right) & =\sum_{\ell} T_{\ell-}(t, r)\left(-\ell(\ell+1) P_{\ell}(\cos \theta)-2 \cot \theta \partial_{\theta} P_{\ell}(\cos \theta)\right),
\end{aligned}
$$

where $P_{\ell}$ are Legendre polynomials. For any tensor $T_{\mu \nu}$, its axial components expanded into (one vector- and one tensortype) axial spherical harmonics at axial symmetry read

$$
\begin{aligned}
& T_{a 3}(t, r, \theta)=\sum_{\ell} T_{\ell a 3}(t, r) \sin \theta \partial_{\theta} P_{\ell}(\cos \theta), \quad a=0,1, \\
& T_{23}(t, r, \theta)=\sum_{\ell} T_{\ell 23}(t, r)\left(-2 \cos \theta \partial_{\theta} P_{\ell}(\cos \theta)-\ell(\ell+1) \sin \theta P_{\ell}(\cos \theta)\right) .
\end{aligned}
$$

Accordingly, in $3+1$ dimensions any vector $V_{\mu}$ can be split into three polar and one axial component. For any vector $V_{\mu}$, its polar components expanded into (one scalar- and one vector-type) polar spherical harmonics at axial symmetry read

$V_{a}(t, r, \theta)=\sum_{\ell} V_{\ell a}(t, r) P_{\ell}(\cos \theta), \quad a=0,1$

$V_{2}(t, r, \theta)=\sum_{\ell} V_{\ell 2}(t, r) \partial_{\theta} P_{\ell}(\cos \theta)$

For any vector $V_{\mu}$ its axial component expanded into (one vector-type) axial spherical harmonics at axial symmetry reads

$$
V_{3}(t, r, \theta)=\sum_{\ell} V_{\ell 3}(t, r) \sin \theta \partial_{\theta} P_{\ell}(\cos \theta)
$$

In what follows, the symbols ${ }^{(i)} h_{\ell \mu \nu},{ }^{(i)} S_{\ell \mu \nu},{ }^{(i)} E_{\ell \mu \nu}$, and $\Delta_{L}{ }^{(i)} h_{\ell} \mu \nu$ appear in expansion of tensors ${ }^{(i)} h_{\mu \nu},{ }^{(i)} S_{\mu \nu},{ }^{(i)} E_{\mu \nu}$, and $\Delta_{L}{ }^{(i)} h_{\mu \nu}$ according to (11)-(16), respectively [cf. (2) and (7)]. The symbols ${ }^{(i)} \zeta_{\ell \mu}$ and ${ }^{(i)} \eta_{\ell \mu}$ appear in expansion of polar gauge vectors ${ }^{(i)} \zeta_{\mu}$ and axial gauge vectors ${ }^{(i)} \eta_{\mu}$ according to (17)-(19), respectively (see below for the usage of these gauge vectors).

\section{POLAR PERTURBATIONS AT AXIAL SYMMETRY}

Although the polar-type perturbations are technically much more involved than axial-type perturbations (in the case of linear Schwarzschild perturbation it took 13 years after solving axial linear perturbations [16] to solve the polar ones [19]), we start the discussion of gravitational perturbations with polar-type perturbations as they are 
indispensable at nonlinear level: even if we start with axialtype perturbations at linear order we end up with polar-type perturbations at second order of perturbation expansion. In axial symmetry for polar perturbations we have

$$
\left({ }^{(i)} h_{\alpha \beta}\right)=\left(\begin{array}{cccc}
{ }^{(i)} h_{00} & { }^{(i)} h_{01} & { }^{(i)} h_{02} & 0 \\
{ }^{(i)} h_{01} & { }^{(i)} h_{11} & { }^{(i)} h_{12} & 0 \\
{ }^{(i)} h_{02} & { }^{(i)} h_{12} & { }^{(i)} h_{22} & 0 \\
0 & 0 & 0 & { }^{(i)} h_{33}
\end{array}\right) \text {, }
$$

with [cf. (11)-(14)]

$$
\begin{aligned}
& { }^{(i)} h_{\ell 00}={ }^{(i)} f_{\ell 00}+2 \partial_{t}{ }^{(i)} \zeta_{\ell 0}-A A^{\prime(i)} \zeta_{\ell 1}, \\
& { }^{(i)} h_{\ell 11}={ }^{(i)} f_{\ell 11}+2 \partial_{r}{ }^{(i)} \zeta_{\ell 1}+\frac{A^{\prime}}{A}{ }^{(i)} \zeta_{\ell 1}, \\
& { }^{(i)} h_{\ell 01}={ }^{(i)} f_{\ell 01}+\partial_{r}{ }^{(i)} \zeta_{\ell 0}+\partial_{t}{ }^{(i)} \zeta_{\ell 1}-\frac{A^{\prime}}{A}{ }^{(i)} \zeta_{\ell 0}, \\
& { }^{(i)} h_{\ell 02}={ }^{(i)} \zeta_{\ell 0}+\partial_{t}{ }^{(i)} \zeta_{\ell 2}, \\
& { }^{(i)} h_{\ell 12}={ }^{(i)} \zeta_{\ell 1}-\frac{2}{r}{ }^{(i)} \zeta_{\ell 2}+\partial_{r}{ }^{(i)} \zeta_{\ell 2}, \\
& { }^{(i)} h_{\ell+}=r^{2(i)} f_{\ell+}+2 r A^{(i)} \zeta_{\ell 1}-\ell(\ell+1)^{(i)} \zeta_{\ell 2}, \\
& { }^{(i)} h_{\ell-}={ }^{(i)} \zeta_{\ell 2},
\end{aligned}
$$

where ${ }^{(i)} \zeta_{\ell 0},{ }^{(i)} \zeta_{\ell 1},{ }^{(i)} \zeta_{\ell 2}$ polar components define the $i$ th order polar gauge vector ${ }^{(i)} \zeta_{\mu}$ [cf. (17) and (18)] and
${ }^{(i)} f_{\ell 00}(t, r),{ }^{(i)} f_{\ell-11}(t, r),{ }^{(i)} f_{\ell 01}(t, r),{ }^{(i)} f_{\ell+}(t, r)$ are Regge-Wheeler variables [16,17], being gauge invariant with respect to gauge transformations induced by ${ }^{(j)} \zeta_{\mu}$ with $j \geq i$, i.e., gauge transformations of the form

$$
\sum_{1 \leq i}{ }^{(i)} h_{\mu \nu} \epsilon^{i} \rightarrow \sum_{1 \leq i}{ }^{(i)} h_{\mu \nu} \epsilon^{i}+\epsilon^{j} \mathcal{L}_{(j)} \bar{g}_{\mu \nu}+\mathcal{O}\left(\epsilon^{j+1}\right) .
$$

Of course, ${ }^{(i)} f_{\ell 00},{ }^{(i)} f_{\ell 11},{ }^{(i)} f_{\ell 01},{ }^{(i)} f_{\ell}+$ so defined are not gauge invariant in general; they change under gauge transformations induced by ${ }^{(j)} \zeta^{\mu}$ with $j<i$ [cf. Bruni et al. [20], Garat and Price [21], Eq. (25)]. The RW gauge corresponds to setting $^{(i)} \zeta_{\ell 0}={ }^{(i)} \zeta_{\ell 1}={ }^{(i)} \zeta_{\ell 2}=0$ in (21)-(27). Other way round, it can be easily seen that starting with any metric perturbation of the form (20), one can put $\left({ }^{(i)} h_{22}-\right.$ $\left.{ }^{(i)} h_{33} / \sin ^{2} \theta\right)$ to zero with a suitable choice of ${ }^{(i)} \zeta_{\ell 2}$, then put ${ }^{(i)} h_{12}$ to zero with a suitable choice of ${ }^{(i)} \zeta_{\ell}$, and finally put ${ }^{(i)} h_{02}$ to zero with a suitable choice of ${ }^{(i)} \zeta_{\ell 0}$ rendering the metric perturbation in RW gauge. At each nonlinear order $(i>1)$ sources have the form

$$
\left({ }^{(i)} S_{\alpha \beta}\right)=\left(\begin{array}{cccc}
{ }^{(i)} S_{00} & { }^{(i)} S_{01} & { }^{(i)} S_{02} & 0 \\
{ }^{(i)} S_{01} & { }^{(i)} S_{11} & { }^{(i)} S_{12} & 0 \\
{ }^{(i)} S_{02} & { }^{(i)} S_{12} & { }^{(i)} S_{22} & 0 \\
0 & 0 & 0 & { }^{(i)} S_{33}
\end{array}\right)
$$

with the components expanded according to (11)-(14). In the following we will use extensively the fact that the sources ${ }^{(i)} S_{\ell \mu \nu}$ fulfill three types of identities:

$$
\begin{aligned}
{ }^{(i)} \mathcal{N}_{\ell 0} & :=\frac{1}{2}\left(\frac{1}{A} \partial_{t}{ }^{(i)} S_{\ell 00}+A \partial_{t}{ }^{(i)} S_{\ell 11}\right)+\frac{1}{r^{2}} \partial_{t}{ }^{(i)} S_{\ell+}-A \partial_{r}{ }^{(i)} S_{\ell 01}-\frac{2 A+r A^{\prime}}{r}{ }^{(i)} S_{\ell 01}+\frac{\ell(\ell+1)}{r^{2}}{ }^{(i)} S_{\ell 02}=0 \\
{ }^{(i)} \mathcal{N}_{\ell 1}: & =\frac{1}{2}\left(\frac{1}{A} \partial_{r}{ }^{(i)} S_{\ell 00}+A \partial_{r}{ }^{(i)} S_{\ell 11}\right)-\frac{1}{r^{2}} \partial_{r}{ }^{(i)} S_{\ell+}-\frac{1}{A} \partial_{t}{ }^{(i)} S_{\ell 01}+\frac{2 A+r A^{\prime}}{r}{ }^{(i)} S_{\ell 11}-\frac{\ell(\ell+1)}{r^{2}}{ }^{(i)} S_{\ell} 12=0 \\
{ }^{(i)} \mathcal{N}_{\ell 2}: & =\frac{1}{2}\left(\frac{1}{A}{ }^{(i)} S_{\ell 00}-A^{(i)} S_{\ell 11}\right)-\frac{1}{A} \partial_{t}{ }^{(i)} S_{\ell 02}+A \partial_{r}{ }^{(i)} S_{\ell 12}+\frac{2 A+r A^{\prime}}{r}{ }^{(i)} S_{\ell 12}-\frac{(\ell-1)(\ell+2)}{r^{2}}{ }^{(i)} S_{\ell-}=0 .
\end{aligned}
$$

These are easily obtained by taking the background divergence of (7): ${ }^{(i)} \mathcal{N}_{\ell \nu}=0$ (with $\nu=0,1,2$ ) follows from (three) polar components of $\bar{\nabla}^{\mu(i)} E_{\mu \nu}=0$. We point out that the authors of $[12,13]$ give different identities: $\bar{\nabla}^{\mu(i)} S_{\mu \nu}=0$ that are apparently not correct in the case of polar-type perturbations, as the (three) polar components of $\bar{\nabla}^{\mu} \Delta_{L}{ }^{(i)} h_{\mu \nu}$ are not identically zero.

The polar components of the system of perturbative Einstein equations (7) are also expanded according to (11)-(14). It is important to note that the gauge degrees of freedom enter ${ }^{(i)} E_{\ell \mu \nu}$ only through the source terms ${ }^{(i)} S_{\ell \mu \nu}$ $\left[{ }^{(i)} S_{\ell \mu \nu}\right.$ depend on gauge functions ${ }^{(j)} \zeta_{\ell 0},{ }^{(j)} \zeta_{\ell 1},{ }^{(j)} \zeta_{\ell 2}$ with $j<i$, that is, on the gauge choices made in previous steps]. On the other hand $\Delta_{L}{ }^{(i)} h_{\ell \mu \nu}$ components are given in terms of Regge-Wheeler gauge invariant variables ${ }^{(i)} f_{\ell} 00,{ }^{(i)} f_{\ell} 11$, ${ }^{(i)} f_{\ell 01},{ }^{(i)} f_{\ell}+$ only [22]. Thus, we solve (7) at order $i$ for Regge-Wheeler variables ${ }^{(i)} f_{\ell 00},{ }^{(i)} f_{\ell 11},{ }^{(i)} f_{\ell 01},{ }^{(i)} f_{\ell+}$ and then recover aAdS gauge with a suitable gauge transformation (see below). It is also known since the Regge and Wheeler seminal paper [16] that it is convenient 
to search for the solutions of the system (7) in terms of one scalar master variable. To achieve it we note that

$$
{ }^{(i)} E_{\ell-}=\frac{1}{4}\left(\frac{1}{A}^{\left({ }^{i}\right)} f_{\ell 00}-A^{(i)} f_{\ell 11}\right)-{ }^{(i)} S_{\ell-}
$$

sets purely algebraic relation between ${ }^{(i)} f_{\ell 00}$ and ${ }^{(i)} f_{\ell 11}$, and the combination

$$
\begin{aligned}
0= & -\frac{1}{2}\left(\frac{1}{A}{ }^{(i)} E_{\ell 00}-A^{(i)} E_{\ell 11}\right)+\frac{1}{A} \partial_{t}^{(i)} E_{\ell 02}-A \partial_{r}{ }^{(i)} E_{\ell 12} \\
& -\frac{2 A+r A^{\prime}}{r}{ }^{(i)} E_{\ell 12}+\frac{(\ell-1)(\ell+2)}{r^{2}}{ }^{(i)} E_{\ell-} \\
\equiv & \frac{1}{2}\left(\frac{1}{A}^{(i)} S_{\ell 00}-A^{(i)} S_{\ell 11}^{(i)}\right)-\frac{1}{A} \partial_{t}^{(i)} S_{\ell 02}+A \partial_{r}{ }^{(i)} S_{\ell 12} \\
& +\frac{2 A+r A^{\prime}}{r}{ }^{(i)} S_{\ell 12}-\frac{(\ell-1)(\ell+2)}{r^{2}}{ }^{(i)} S_{\ell-}
\end{aligned}
$$

reduces to the identity (32) fulfilled by the sources. Thus, after eliminating ${ }^{(i)} f_{\ell}$ on from (33) we are left with three RW gauge invariant potentials ${ }^{(i)} f_{\ell 11},{ }^{(i)} f_{\ell 01},{ }^{(i)} f_{\ell}+$ to satisfy five linearly independent equations: ${ }^{(i)} E_{\ell 00}={ }^{(i)} E_{\ell 01}=$ ${ }^{(i)} E_{\ell+}={ }^{(i)} E_{\ell 02}={ }^{(i)} E_{\ell 12}=0$. All formulas presented so far hold for any spherically symmetric solution of vacuum Einstein equations [i.e., Schwarzschild and Schwarzschild(anti-)de Sitter] in Schwarzschild-like coordinates (10) with $A=1 \pm\left(r^{2} / \mathfrak{l}^{2}\right)-2 M / r$. Being interested in perturbations of AdS space, from now on we restrict to maximally symmetric solutions $(M=0)$, as in this case apart form the general identity $r^{2} A^{\prime \prime}=2 A-2$ we have also an identity $r A^{\prime}=2 A-2$ (valid only in $M=0$ case) and it slightly simplifies discussion that follows. We introduce the master scalar variable for polar perturbations at linear order in such a way, to make the equations ${ }^{(1)} E_{\ell 02}={ }^{(1)} E_{\ell} 12=0$ identically satisfied. Namely, writing down the potentials ${ }^{(1)} f_{\ell 11},{ }^{(1)} f_{\ell 01},{ }^{(1)} f_{\ell}+$ at linear order in terms of linear combination of the derivatives (up to second order) of one master scalar variable for polar perturbations ${ }^{(1)} \Phi_{\ell}^{\mathcal{P}}$, and then plugging this combination into ${ }^{(1)} E_{\ell 02}={ }^{(1)} E_{\ell 12}=0$ yields a unique such combination (up to a multiplicative factor):

$$
\begin{aligned}
{ }^{(1)} f_{\ell 00} & =\frac{r}{2}\left(\partial_{t t}{ }^{(1)} \Phi_{\ell}^{\mathcal{P}}+A^{2} \partial_{r r}{ }^{(1)} \Phi_{\ell}^{\mathcal{P}}\right)+A^{2} \partial_{r}{ }^{(1)} \Phi_{\ell}^{\mathcal{P}} \\
& =A^{2(1)} f_{\ell} 11
\end{aligned}
$$

This is an easy way to recover old results of Mukohyama [18], Eq. (44) and Kodama and Ishibashi [23], Eq. (3.14). At linear order, the three remaining equations ${ }^{(1)} E_{\ell 00}=$ ${ }^{(1)} E_{\ell 01}={ }^{(1)} E_{\ell+}=0$ are satisfied if and only if ${ }^{(1)} \Phi_{\ell}^{\mathcal{P}}$ solves the homogeneous radial wave equation [24]:

$$
\begin{aligned}
\tilde{\square}_{\ell}{ }^{(1)} \Phi_{\ell}^{\mathcal{P}}:= & \frac{1}{A} \partial_{t t}{ }^{(1)} \Phi_{\ell}^{\mathcal{P}}-A \partial_{r r}{ }^{(1)} \Phi_{\ell}^{\mathcal{P}}-A^{\prime} \partial_{r}{ }^{(1)} \Phi_{\ell}^{\mathcal{P}} \\
& +\frac{\ell(\ell+1)}{r^{2}}{ }^{(1)} \Phi_{\ell}^{\mathcal{P}}=0,
\end{aligned}
$$

namely, under the substitution (35)-(37)

$$
\begin{aligned}
{ }^{(1)} E_{\ell 00} & =\frac{A^{2}}{4}\left(\frac{1}{A^{2}} \partial_{t t}+r \partial_{r r}+\partial_{r}+\frac{6}{r}\right) \tilde{\square}_{\ell}{ }^{(1)} \Phi_{\ell}^{\mathcal{P}}, \\
{ }^{(1)} E_{\ell 01} & =\left(\frac{r}{2} \partial_{r}+1+\frac{1}{2 A}\right) \partial_{t} \tilde{\square}_{\ell}{ }^{(1)} \Phi_{\ell}^{\mathcal{P}}, \\
{ }^{(1)} E_{\ell+} & =\left(-\frac{r^{3}}{4 A} \partial_{t t}+\frac{r^{3} A}{4} \partial_{r r}+r^{2}\left(2 A-\frac{1}{2}\right) \partial_{r}+r\left(\frac{5}{2} A-1\right)\right) \tilde{\square}_{\ell}{ }^{(1)} \Phi_{\ell}^{\mathcal{P}} .
\end{aligned}
$$

To proceed to higher orders it is convenient to invert (35)-(37) for the master scalar variable ${ }^{(1)} \Phi_{\ell}^{\mathcal{P}}$. We take the following linear combination of RW gauge invariant potentials ${ }^{(i)} f_{\ell 11},{ }^{(i)} f_{\ell 01},{ }^{(i)} f_{\ell}+$ and their first derivatives:

$$
{ }^{(i)} \Phi_{\ell}^{\mathcal{P}}=\frac{2 r}{\ell(\ell+1)}\left({ }^{(i)} f_{\ell+}+2 A \frac{A^{(i)} f_{\ell 11}-r \partial_{r}{ }^{(i)} f_{\ell+}}{(\ell-1)(\ell+2)}\right) .
$$

Indeed at linear order, under substitution (35)-(37) with (38) fulfilled (42) becomes an identity. If translated to the asymptotically flat Schwarzschild case, this definition would corresponds to Brizuela et al. [25], Eq. (11), which dates back to the choice made by Moncrief [26]. We take (42) as a definition of the master scalar variable also at higher (nonlinear) orders. This definition determines the source for the inhomogeneous wave equation for master scalar variable at higher orders, namely, substituting (42) on the rhs of (38) we find that 


$$
\begin{aligned}
\tilde{\square}_{\ell}^{(i)} \Phi_{\ell}^{\mathcal{P}}= & \frac{4 r^{2}}{(\ell-1) \ell(\ell+1)(\ell+2)} \\
& \times\left((A / r)\left(A \Delta_{L}{ }^{(i)} h_{\ell} 11-\frac{1}{A} \Delta_{L}{ }^{(i)} h_{\ell} 00\right)+\frac{(\ell-1)(\ell+2)-2(3 A-2)}{r^{3}} \Delta_{L}{ }^{(i)} h_{\ell+}-2 A \partial_{r}\left(\Delta_{L}{ }^{(i)} h_{\ell+} / r^{2}\right)\right. \\
& \left.-\frac{2 \ell(\ell+1)}{r^{2}} A_{L}{ }^{(i)} h_{\ell 12}+\frac{(\ell-1) \ell(\ell+1)(\ell+2)}{r^{3}} \Delta_{L}{ }^{(i)} h_{\ell-}\right) .
\end{aligned}
$$

Thus, at higher orders $(i \geq 2)$ the definition (42) leads to

$$
{ }^{(i)} \mathcal{B}_{\ell}^{\mathcal{P}}:=\tilde{\square}_{\ell}{ }^{(i)} \Phi_{\ell}^{\mathcal{P}}-{ }^{(i)} \tilde{S}_{\ell}^{\mathcal{P}}=0,
$$

with

$$
\begin{aligned}
{ }^{(i)} \tilde{S}_{\ell}^{\mathcal{P}}= & \frac{4 r^{2}}{(\ell-1) \ell(\ell+1)(\ell+2)} \\
& \times\left((A / r)\left(A^{(i)} S_{\ell 11}-\frac{1}{A}{ }^{(i)} S_{\ell 00}\right)+\frac{(\ell-1)(\ell+2)-2(3 A-2)}{r^{3}}{ }^{(i)} S_{\ell+}-2 A \partial_{r}\left({ }^{(i)} S_{\ell+} / r^{2}\right)\right. \\
& \left.-\frac{2 \ell(\ell+1)}{r^{2}} A^{(i)} S_{\ell 12}+\frac{(\ell-1) \ell(\ell+1)(\ell+2)}{r^{3}}{ }^{(i)} S_{\ell-}\right)
\end{aligned}
$$

for $\ell \geq 2$ (here the $\ell=0$ and $\ell=1$ cases have to be treated separately). The linear rules (35)-(37) are generalized to

$$
\begin{aligned}
& { }^{(i)} f_{\ell 00}=A^{2}\left(\frac{r}{2}\left(\frac{1}{A^{2}} \partial_{t t}{ }^{(i)} \Phi_{\ell}^{\mathcal{P}}+\partial_{r r}{ }^{(i)} \Phi_{\ell}^{\mathcal{P}}\right)+\partial_{r}{ }^{(i)} \Phi_{\ell}^{\mathcal{P}}+{ }^{(i)} \alpha_{\ell}(t, r)\right)+4 A^{(i)} S_{\ell-}, \\
& { }^{(i)} f_{\ell-11}=\frac{r}{2}\left(\frac{1}{A^{2}} \partial_{t t}{ }^{(i)} \Phi_{\ell}^{\mathcal{P}}+\partial_{r r}{ }^{(i)} \Phi_{\ell}^{\mathcal{P}}\right)+\partial_{r}{ }^{(i)} \Phi_{\ell}^{\mathcal{P}}+{ }^{(i)} \alpha_{\ell}(t, r), \\
& { }^{(i)} f_{\ell 01}=r \partial_{r t}{ }^{(i)} \Phi_{\ell}^{\mathcal{P}}+A^{-1} \partial_{t}{ }^{(i)} \Phi_{\ell}^{\mathcal{P}}+{ }^{(i)} \beta_{\ell}(t, r), \\
& { }^{(i)} f_{\ell+}=-\frac{r}{2}\left(\frac{1}{A} \partial_{t t}{ }^{(i)} \Phi_{\ell}^{\mathcal{P}}-A \partial_{r r}{ }^{(i)} \Phi_{\ell}^{\mathcal{P}}\right)+(2 A-1) \partial_{r}{ }^{(i)} \Phi_{\ell}^{\mathcal{P}}+{ }^{(i)} \gamma_{\ell}(t, r),
\end{aligned}
$$

with the set of three functions ${ }^{(i)} \alpha_{\ell},{ }^{(i)} \beta_{\ell}$, and ${ }^{(i)} \gamma_{\ell}$ for each $\ell$, which still have to be specified. These functions are set in such a way that the equations ${ }^{(i)} E_{\ell 02}={ }^{(i)} E_{\ell 12}=0$ are identically satisfied, while the equations ${ }^{(i)} E_{\ell 00}={ }^{(i)} E_{\ell 01}=$ ${ }^{(i)} E_{\ell+}=0$ are satisfied if and only if ${ }^{(i)} \Phi_{\ell}^{\mathcal{P}}$ solves the inhomogeneous wave equation (44), i.e.,

$$
\begin{aligned}
{ }^{(i)} E_{\ell 00} & =\frac{A^{2}}{4}\left(\frac{r}{A^{2}} \partial_{t t}+r \partial_{r r}+\partial_{r}+\frac{6}{r}\right){ }^{(i)} \mathcal{B}_{\ell}^{\mathcal{P}}, \\
{ }^{(i)} E_{\ell 01} & =\left(\frac{r}{2} \partial_{r}+1+\frac{1}{2 A}\right) \partial_{t}{ }^{(i)} \mathcal{B}_{\ell}^{\mathcal{P}}, \\
{ }^{(i)} E_{\ell+} & =\left(-\frac{r^{3}}{4 A} \partial_{t t}+\frac{r^{3} A}{4} \partial_{r r}+r^{2}\left(2 A-\frac{1}{2}\right) \partial_{r}+r\left(\frac{5}{2} A-1\right)\right){ }^{(i)} \mathcal{B}_{\ell}^{\mathcal{P}},
\end{aligned}
$$

cf. the linear case (39)-(41). It is worth noting that due to the identities (30)-(32) the solutions for ${ }^{(i)} \alpha_{\ell},{ }^{(i)} \beta_{\ell}$ and ${ }^{(i)} \gamma_{\ell}$ can be found in a purely algebraic way, without the need to solve any (partial) differential equations. The results read

$$
\begin{aligned}
{ }^{(i)} \alpha_{\ell}= & -\frac{2 r^{2}\left(\frac{1}{A}{ }^{(i)} S_{\ell 00}-A^{(i)} S_{\ell 11}\right)-\frac{r^{2}}{A}(\ell-1)(\ell+2)\left(\frac{1}{A}{ }^{(i)} S_{\ell 00}+A^{(i)} S_{\ell 11}\right)+4 \partial_{r}\left(r^{(i)} S_{\ell+}\right)+\frac{4 r^{3}}{A} \partial_{t}{ }^{(i)} S_{\ell 01}}{(\ell-1) \ell(\ell+1)(\ell+2)} \\
& -\frac{2}{A} S_{\ell-},
\end{aligned}
$$




$$
\begin{aligned}
{ }^{(i)} \beta_{\ell} & =\frac{r \ell(\ell+1)^{(i)} S_{\ell 02}-r^{2}(4 A-1-\ell(\ell+1) / 2)^{(i)} S_{\ell 01}-r^{3} A \partial_{r}{ }^{(i)} S_{\ell 01}}{(\ell-1) \ell(\ell+1)(\ell+2) / 4}, \\
{ }^{(i)} \gamma_{\ell} & =-\frac{r^{2(i)} S_{\ell 00}+r A \ell(\ell+1)^{(i)} S_{\ell 12}+(2 A-1-\ell(\ell+1) / 2)^{(i)} S_{\ell+}+r A \partial_{r}{ }^{(i)} S_{\ell+}+2^{(i)} S_{\ell-\cdot}}{(\ell-1) \ell(\ell+1)(\ell+2) / 4}
\end{aligned}
$$

We stress that the Regge-Wheeler gauge [i.e., setting ${ }^{(i)} \zeta_{\ell 0}={ }^{(i)} \zeta_{\ell 1}={ }^{(i)} \zeta_{\ell 2}=0$ in (21)-(27)] is not aAdS (nor asymptotically flat in the corresponding $\Lambda=0$ case). The asymptotic form of vacuum metric perturbations corresponding to an aAdS space was found in [27]. Here we follow the discussion in Sec. II.C of [28]. We ensure that $O(2,3)$ is an asymptotic symmetry of $g_{\mu \nu}=\bar{g}_{\mu \nu}+\delta g_{\mu \nu}$ by requiring the Killing equation to be satisfied in an asymptotic sense

$$
\mathcal{L}_{\xi} g_{\mu \nu}=\mathcal{O}\left(\delta g_{\mu \nu}\right)
$$

for all Killing vectors of AdS space. Thus, we are looking for an asymptotic form of metric perturbations $\delta g_{\mu \nu} \sim$ $1 / r^{\gamma_{\mu \nu}}$, such that this asymptotic form is preserved by coordinate transformations generated by any AdS Killing vector $\xi$. This holds for

$\gamma_{r r}=5, \quad \gamma_{r \mu}=4$ and $\gamma_{\mu \nu}=1 \quad$ for $\mu, \nu \neq r$

(in $3+1$ dimensions) [27,28]. The asymptotic behavior of solutions of homogeneous wave equation $\tilde{\square}_{\ell} \Phi_{\ell}=0$ (38) reads

$$
\Phi_{\ell} \sim a_{\ell}+\frac{b_{\ell}}{r}+\mathcal{O}\left(\frac{1}{r^{2}}\right),
$$

thus, already at linear order RW variables reconstructed from (35)-(37) do not have AdS asymptotics and the RW gauge is not aAdS. This is not a problem as long as a suitable gauge transformation [i.e., suitable functions $\left.{ }^{(i)} \zeta_{\ell 0},{ }^{(i)} \zeta_{\ell 1},{ }^{(i)} \zeta_{\ell 2}\right]$ to render metric perturbation ${ }^{(i)} h_{\mu \nu}$ in aAdS form can be found. The necessary condition for such a gauge transformation to exist for polar-type perturbations is $b_{\ell} \equiv 0$ in (58); that is, the correct boundary condition at infinity for master scalar variable for polar perturbations ${ }^{(i)} \Phi_{\ell}^{\mathcal{P}}$ reads

$$
{ }^{(i)} \Phi_{\ell}^{\mathcal{P}}={ }^{(i)} a_{\ell}+\mathcal{O}\left(\frac{1}{r^{2}}\right)
$$

(this correct form of asymptotics was stressed for the first time in [12]). After separating time dependence in (44) in the form ${ }^{(i)} \Phi_{\ell}^{\mathcal{P}}(t, r)=\sum_{k}{ }^{(i)} \Phi_{\ell k}^{\mathcal{P}}(r) \cos \left(\omega_{k} t+\phi_{k}\right)$ (with some suitable set of frequencies $\omega_{k}$ ) we end up with ordinary second order differential equations for ${ }^{(i)} \Phi_{\ell k}^{\mathcal{P}}(r)$ coefficients. Thus, to obtain a unique solution we need two boundary conditions; these are smoothness in the center and (59). To get the asymptotics of the sources ${ }^{(i)} S_{\ell}^{\mathcal{P}}$ in (44) compatible with the asymptotics of the $\tilde{\square}_{\ell}$ operator (58) (to allow for an easy control over aAdS asymptotics step after step in perturbation expansion) it is necessary to keep metric perturbations in aAdS form at each order. Once the boundary condition (59) is satisfied the aAdS gauge can be recovered with the polar gauge vector induced by [cf. (17) and (18)]

${ }^{(i)} \zeta_{\ell 1}=\frac{1}{4}\left(\mathfrak{l}^{2} A^{-1} \partial_{t t}{ }^{(i)} \Phi_{\ell}^{\mathcal{P}}-4 r \partial_{r}{ }^{(i)} \Phi_{\ell}^{\mathcal{P}}-r^{2} \partial_{r r}{ }^{(i)} \Phi_{\ell}^{\mathcal{P}}\right)$,

${ }^{(i)} \zeta_{\ell 0}=-r \partial_{t}{ }^{(i)} \zeta_{\ell 1}+\frac{r}{3 A} \partial_{t}\left(\mathfrak{l}^{2} \partial_{t t}{ }^{(i)} \Phi_{\ell}^{\mathcal{P}}+{ }^{(i)} \Phi_{\ell}^{\mathcal{P}}\right)$,

${ }^{(i)} \zeta_{\ell 2}=\frac{r}{3}{ }^{(i)} \zeta_{\ell 1}$

In fact at first and second order we found also somewhat simpler rules:

$$
\begin{aligned}
& { }^{(1)} \zeta_{\ell 1}=\frac{1}{4}\left(\mathfrak{l}^{2} A^{-1} \partial_{t t}{ }^{(1)} \Phi_{\ell}^{\mathcal{P}}-r \partial_{r}{ }^{(1)} \Phi_{\ell}^{\mathcal{P}}\right), \\
& { }^{(1)} \zeta_{\ell 0}=-r \partial_{t}{ }^{(1)} \zeta_{\ell 1}+\frac{r}{3 A} \partial_{t}\left(\mathfrak{l}^{2} \partial_{t t}{ }^{(1)} \Phi_{\ell}^{\mathcal{P}}+{ }^{(1)} \Phi_{\ell}^{\mathcal{P}}\right), \\
& { }^{(1)} \zeta_{\ell 2}=\frac{r}{3}{ }^{(1)} \zeta_{\ell 1},
\end{aligned}
$$

and

$$
\begin{aligned}
& { }^{(2)} \zeta_{\ell 0}=0 \\
& { }^{(2)} \zeta_{\ell 1}=\frac{1}{2}\left({ }^{(2)} \Phi_{\ell}^{\mathcal{P}}-\frac{1}{2} \partial_{r r}\left(r^{2}{ }^{(2)} \Phi_{\ell}^{\mathcal{P}}\right)\right), \\
& { }^{(2)} \zeta_{\ell 2}=\frac{r}{3}{ }^{(2)} \zeta_{\ell 1} .
\end{aligned}
$$

To summarize: to satisfy the set of perturbative Einstein equations (7) for polar-type perturbations at axial symmetry, at any nonlinear order, for angular momenta $\ell \geq 2$ [cf. (11)-(14)], it is enough to solve just one inhomogeneous wave equation for a scalar master variable for polar perturbations (44) with the source term given in (45), and then reconstruct the Regge-Wheeler gauge invariant potentials ${ }^{(i)} f_{\ell 00},{ }^{(i)} f_{\ell 11},{ }^{(i)} f_{\ell 01}$ and ${ }^{(i)} f_{\ell}+$ according to (46)-(49) together with (53)-(55). Then with the gauge 
transformation (60)-(62) the resulting perturbations ${ }^{(i)} h_{\ell} \mu \nu$ [cf. (21)-(27)] can be put in asymptotically AdS form.

As was stressed above the special cases $\ell=0$ and $\ell=1$ need a special treatment.

\section{A. The $\ell=0$ case for polar perturbations}

In the $\ell=0$ case, the only nontrivial equations are $0=$ ${ }^{(i)} E_{0+}={ }^{(i)} E_{001}={ }^{(i)} E_{01 \pm 0}:=\left(A^{(i)} E_{011} \pm(1 / A)^{(i)} E_{000}\right)$. In fact

$$
\begin{gathered}
{ }^{(i)} E_{01-0}-\frac{1}{r} \partial_{r}{ }^{(i)} E_{0+}+\frac{r}{2} \partial_{r}{ }^{(i)} E_{01+0}-\frac{r}{A} \partial_{t}{ }^{(i)} E_{001}+\frac{2 A-1}{A}{ }^{(i)} E_{01+0} \\
\partial_{t}\left({ }^{(i)} E_{0+}+\frac{r^{2}}{2}{ }^{(i)} E_{01+0}\right)-2 r(2 A-1)^{(i)} E_{001}-r^{2} A \partial_{r}{ }^{(i)} E_{001} \equiv 0
\end{gathered}
$$

[due to identities (30) and (31)], and ${ }^{(i)} f_{001},{ }^{(i)} f_{0}$ + become gauge degrees of freedom [i.e., they can be put to zero with a suitable choice of ${ }^{(i)} \zeta_{00}$ and ${ }^{(i)} \zeta_{01}$ ]. Thus, we are left with two equations $0={ }^{(i)} E_{001}={ }^{(i)} E_{01+0}$ for two unknown functions ${ }^{(i)} f_{000}$ and ${ }^{(i)} f_{011}$, where ${ }^{(i)} f_{001}$ and ${ }^{(i)} f_{0+}$ can be freely specified and we put them to zero. This system can be easily integrated to yield

$$
\begin{aligned}
& { }^{(i)} f_{011}={ }^{(i)} f_{0}^{\mathcal{P} \text { res }}+r A^{-1} \int{ }^{t}{ }^{(i)} S_{001} d t^{\prime} \\
& { }^{(i)} f_{000}=A^{2(i)} f_{011}-A \int_{\infty}^{r} r^{\prime}\left({ }^{(i)} S_{011}+\frac{1}{A^{2}}{ }^{(i)} S_{000}\right) d r^{\prime},
\end{aligned}
$$

with the residual degree of freedom ${ }^{(i)} f_{0}^{\mathcal{P}}{ }^{\text {res }} \equiv{ }^{(i)} f_{0}^{\mathcal{P} \text { res }}(r)$ that is not set by the equations $0=E_{001}^{(i)}=E_{01+0}^{(i)}$. It is, however, uniquely determined as the solution of the first order ordinary differential equation set by (the time independent part of) the equation ${ }^{(i)} E_{0+}=0$. The solution reads

$$
{ }^{(i)} f_{0}^{\mathcal{P} \text { res }}=\frac{1}{r A^{2}} \int_{0}^{r}\left[\frac{r^{\prime 2}}{2}\left(\frac{1}{A}{ }^{(i)} \bar{S}_{000}+A^{(i)} \bar{S}_{011}\right)+{ }^{(i)} \bar{S}_{0+}\right] d r^{\prime}
$$

where ${ }^{(i)} \bar{S}_{\ell \mu \nu}$ are time independent parts of ${ }^{(i)} S_{\ell \mu \nu}$ and ${ }^{(i)} f_{0}^{\mathcal{P} \text { res }}$ is thus by definition time independent. At linear order the $\ell=0$ part of the perturbation reduces to a pure gauge, as expected [as ${ }^{(1)} S_{\mu \nu} \equiv 0$ ]. Moreover, for $i>1,{ }^{(i)} f_{000}$ and ${ }^{(i)} f_{011}$ satisfy aAdS conditions (56) and (57), thus no further gauge transformation is needed.

\section{B. The $\ell=1$ case for polar perturbations}

In the $\ell=1$ case we proceed similarly to the $\ell=0$ case. There are six nontrivial Einstein equations: $0={ }^{(i)} E_{1+}=$ ${ }^{(i)} E_{101}={ }^{(i)} E_{102}={ }^{(i)} E_{112}={ }^{(i)} E_{11 \pm 0}:=\left(A^{(i)} E_{111} \pm(1 / A)^{(i)} E_{100}\right)$. In fact

$$
\begin{array}{r}
\frac{1}{2}{ }^{(i)} E_{11-0}+\frac{1}{A} \partial_{t}{ }^{(i)} E_{102}-A \partial_{r}{ }^{(i)} E_{112}-\frac{2}{r}(2 A-1)^{(i)} E_{112} \equiv 0 \\
\partial_{t}\left({ }^{(i)} E_{1+}+\frac{r^{2}}{2}{ }^{(i)} E_{11+0}\right)-2 r(2 A-1)^{(i)} E_{101}-r^{2} A \partial_{r}{ }^{(i)} E_{101}+2^{(i)} E_{102} \equiv 0 \\
-\frac{1}{r} \partial_{r}{ }^{(i)} E_{1+}+\frac{r}{2} \partial_{r}{ }^{(i)} E_{11+0}+\frac{2 A-1}{A}{ }^{(i)} E_{11+0}-\frac{r}{A} \partial_{t}^{(i)} E_{101}-\frac{2}{r}{ }^{(i)} E_{112}+{ }^{(i)} E_{11-0} \equiv 0
\end{array}
$$

due to identities (30)-(32), and ${ }^{(i)} f_{1}$ - becomes gauge degree of freedom [i.e., it can be put to zero with a suitable choice of ${ }^{(i)} \zeta_{11}$ in function of $\left.{ }^{(i)} \zeta_{12}\right]$. We set ${ }^{(i)} f_{1+}$ to zero and integrate in sequence the equations $0=r^{(i)} E_{101}+2^{(i)} E_{102}=$ ${ }^{(i)} E_{1+}+\left(r^{2} / 2\right)^{(i)} E_{11+0}={ }^{(i)} E_{112}$ to get 


$$
\begin{aligned}
& { }^{(i)} f_{101}=\frac{1}{r A^{1 / 2}} \int_{0}^{r} \frac{r^{\prime}}{A^{1 / 2}}\left(r^{\prime(i)} S_{101}+2^{(i)} S_{102}\right) d r^{\prime}, \\
& { }^{(i)} f_{111}=\frac{1}{r^{2} A^{3 / 2}} \int_{0}^{r} \frac{r^{\prime}}{A^{1 / 2}}\left[\frac{r^{\prime 2}}{2}\left(\frac{1}{A}{ }^{(i)} S_{100}+A S_{111}\right)+{ }^{(i)} S_{1+}\right] d r^{\prime}, \\
& { }^{(i)} f_{100}=r A^{1 / 2} \int_{\infty}^{r} \frac{A^{1 / 2}}{r^{\prime}}\left(2^{(i)} S_{112}+\frac{1}{A} \partial_{t}{ }^{(i)} f_{101}-\frac{2 A-1}{r^{\prime}}{ }^{(i)} f_{111}\right) d r^{\prime},
\end{aligned}
$$

respectively. Then equations $0={ }^{(i)} E_{101}={ }^{(i)} E_{1}+$ are also satisfied due to identities (30)-(32). Alternatively, one can integrate the ${ }^{(i)} E_{101}=0$ equation [instead of the ${ }^{(i)} E_{1+}+\left(r^{2} / 2\right)^{(i)} E_{11+0}=0$ equation] up to a residual ${ }^{(i)} f_{1}^{\mathcal{P} \text { res }} \equiv{ }^{(i)} f_{1}^{\mathcal{P} \text { res }}(r)$ :

$$
{ }^{(i)} f_{111}^{(i)}={ }^{(i)} f_{1}^{\mathcal{P} \text { res }}+\frac{1}{A} \int^{t}\left(r^{(i)} S_{101}-\frac{1}{r}{ }^{(i)} f_{101}\right) d t^{\prime}
$$

and then set ${ }^{(i)} f_{1}^{\mathcal{P}}$ res by (the time independent part of) the equation ${ }^{(i)} E_{1+}^{(i)}+\left(r^{2} / 2\right)^{(i)} E_{11+0}=0$ :

$$
{ }^{(i)} f_{1}^{\mathcal{P} \text { res }}=\frac{1}{r^{2} A^{3 / 2}} \int_{0}^{r} \frac{r^{\prime}}{A^{1 / 2}}\left[\frac{r^{\prime 2}}{2}\left(\frac{1}{A}{ }^{(i)} \bar{S}_{100}+A^{(i)} \bar{S}_{111}\right)+{ }^{(i)} \bar{S}_{1+}\right] d r^{\prime} \text {. }
$$

Then the equations $0={ }^{(i)} E_{1+}={ }^{(i)} E_{11+0}$ are also satisfied due to identities (30)-(32). This is a bit more convenient in some practical applications, as for the sources generated by time-periodic solutions, taking integrals in time is straightforward. At linear order the $\ell=1$ part of the perturbation reduces to a pure gauge, as expected [as $\left.{ }^{(1)} S_{\mu \nu} \equiv 0\right]$. Moreover for $i>1,{ }^{(i)} f_{101},{ }^{(i)} f_{100}$, and (i) $f_{111}$ satisfy aAdS condition (56) and (57); thus, no further gauge transformation is needed.

\section{AXIAL PERTURBATIONS AT AXIAL SYMMETRY}

The axial-type perturbations can be treated along the same lines as polar-type perturbations discussed in the previous section, but are in fact much easier to deal with at a technical level. We describe axial-type perturbations in this section for the sake of completeness. In axial symmetry for axial-type perturbations we have

$$
\left({ }^{(i)} h_{\alpha \beta}\right)=\left(\begin{array}{cccc}
0 & 0 & 0 & { }^{(i)} h_{03} \\
0 & 0 & 0 & { }^{(i)} h_{13} \\
0 & 0 & 0 & { }^{(i)} h_{23} \\
{ }^{(i)} h_{03} & { }^{(i)} h_{13} & { }^{(i)} h_{23} & 0
\end{array}\right),
$$

with [cf. (15) and (16)]

$$
\begin{aligned}
& { }^{(i)} h_{\ell 03}={ }^{(i)} f_{\ell 03}+\partial_{t}{ }^{(i)} \eta_{\ell}, \\
& { }^{\left({ }^{(i)}\right.} h_{\ell 13}={ }^{(i)} f_{\ell 13}+\partial_{r}{ }^{(i)} \eta_{\ell}-2^{(i)} \eta_{\ell} / r \\
& { }^{(i)} h_{\ell 23}={ }^{(i)} \eta_{\ell},
\end{aligned}
$$

where an axial component ${ }^{(i)} \eta_{\ell}(t, r)$ defines the $i$ th order axial gauge vector ${ }^{(i)} \eta_{\mu}$ [cf. (19)] and ${ }^{(i)} f_{\ell 03}(t, r)$, ${ }^{(i)} f_{\ell 13}(t, r)$ are Regge-Wheeler variables [16,17] being gauge invariant with respect to gauge transformations induced by ${ }^{(j)} \eta_{\mu}$ with $j \geq i$; cf. (28). The sources and Einstein equations (7) read accordingly:

$$
\left({ }^{(i)} S_{\alpha \beta}\right)=\left(\begin{array}{cccc}
0 & 0 & 0 & { }^{(i)} S_{03} \\
0 & 0 & 0 & { }^{(i)} S_{13} \\
0 & 0 & 0 & { }^{(i)} S_{23} \\
{ }^{(i)} S_{03} & { }^{(i)} S_{13} & { }^{(i)} S_{23} & 0
\end{array}\right),
$$

with the components expanded according to (15) and (16). In the axial case, the sources fulfill the following identity:

$$
\begin{aligned}
{ }^{(i)} \mathcal{N}_{\ell 3}:= & -\frac{1}{A} \partial_{t}{ }^{(i)} S_{\ell 03}+A \partial_{r}{ }^{(i)} S_{\ell 13}+\frac{2}{r}(2 A-1)^{(i)} S_{\ell 13} \\
& -\frac{(\ell-1)(\ell+2)}{r^{2}}{ }^{(i)} S_{\ell 23}=0 .
\end{aligned}
$$

This is again obtained by taking the background divergence of (7): ${ }^{(i)} \mathcal{N}_{\ell 3}=0$ follows from the (one) axial component of $\bar{\nabla}^{\mu(i)} E_{\mu \nu}=0$ [the (one) axial component of $\bar{\nabla}^{\mu} \Delta_{L}{ }^{(i)} h_{\mu \nu}$ vanishes identically thus the (one) axial component of $\bar{\nabla}^{\mu(i)} S_{\mu \nu}$ vanishes as well and (only) in that sense the formula $\bar{\nabla}^{\mu(i)} S_{\mu \nu}=0$ in [12] is correct for axial perturbations]. Similarly to the polar case, the gauge degrees of freedom enter ${ }^{(i)} E_{\ell \mu \nu}$ only through the source terms ${ }^{(i)} S_{\ell \mu \nu}$ [and ${ }^{(i)} S_{\ell \mu \nu}$ depend on gauge functions 
${ }^{(j)} \eta_{\ell}$ with $\left.j<i\right]$. Thus, we solve (7) at order $i$ for ReggeWheeler variables ${ }^{(i)} f_{\ell 03},{ }^{(i)} f_{\ell 13}$ and then recover the aAdS gauge with a suitable gauge transformation (see below). In the axial case, the perturbative Einstein equations can be easily integrated and the definition of the master scalar variable is straightforward. We set

$$
{ }^{(i)} f_{\ell 13}=\frac{r}{A}{ }^{(i)} \Phi_{\ell}^{\mathcal{A}} \text {. }
$$

For $\ell \geq 2$ the three perturbative Einstein equations ${ }^{(i)} E_{\ell 03},{ }^{(i)} E_{\ell 13}$, and ${ }^{(i)} E_{\ell 23}$ can be combined as follows: [the $\ell=1$ case has to be treated separately, as $\left.{ }^{(i)} E_{123} \equiv 0\right]$. First,

$$
\begin{aligned}
0 & =-\frac{1}{A} \partial_{t}{ }^{(i)} E_{\ell 03}+A \partial_{r}{ }^{(i)} E_{\ell 13}+\frac{2}{r}(2 A-1)^{(i)} E_{\ell} 13 \\
& -\frac{(\ell-1)(\ell+2)}{r^{2}}{ }^{(i)} E_{\ell 23}
\end{aligned}
$$

reduces to the identity (87). Second,

$0=\frac{2 A}{r}\left({ }^{(i)} E_{\ell 13}-\partial_{r}{ }^{(i)} E_{\ell 23}\right)+\frac{4}{r^{2}}{ }^{(i)} E_{\ell 23} \equiv \tilde{\square}_{\ell}{ }^{(i)} \Phi_{\ell}^{\mathcal{A}}-{ }^{(i)} \tilde{S}_{\ell}^{\mathcal{A}}$,

where the $\tilde{\square}_{\ell}$ operator was defined in (38) and the axial source ${ }^{(i)} S_{\ell}^{\mathcal{A}}$ reads

$$
\left.{ }^{(i)} \tilde{S}_{\ell}^{\mathcal{A}}=\frac{2 A}{r}{ }^{\left({ }^{(i)}\right.} S_{\ell 13}-\partial_{r}{ }^{(i)} S_{\ell 23}\right)+\frac{4}{r^{2}}{ }^{(i)} S_{\ell 23} .
$$

Finally,

$$
2 A^{(i)} E_{\ell 23}=-\partial_{t}^{(i)} f_{\ell 03}+A \partial_{r}\left(A^{(i)} f_{\ell 13}\right)-2 A^{(i)} S_{\ell 23}=0
$$

can be easily integrated in time for ${ }^{(i)} f_{\ell 03}$. Similarly to the polar case RW gauge [i.e., ${ }^{(i)} \eta_{\ell} \equiv 0$ in (83)-(85)] is not aAdS and the necessary condition, for a gauge transformation to aAdS gauge to exist, is $a_{\ell} \equiv 0$ in (58), that is, the correct boundary condition at infinity for a master scalar variable for axial perturbations ${ }^{(i)} \Phi_{\ell}^{\mathcal{A}}$ reads

$$
{ }^{(i)} \Phi_{\ell}^{\mathcal{A}}=\frac{{ }^{(i)} b_{\ell}}{r}+\mathcal{O}\left(\frac{1}{r^{3}}\right)
$$

Once the boundary condition (93) is satisfied the aAdS gauge can be recovered with the axial gauge vector induced by [cf. (19)]

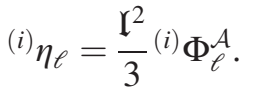

To summarize, to satisfy the set of perturbative Einstein equations (7) for axial-type perturbations at axial symmetry, at any nonlinear order, for angular momenta $\ell \geq 2$ [cf. (15) and (16)], it is enough to solve just one inhomogeneous wave equation for the scalar master variable for axial perturbations (90) with the source term given in (91), and then obtain the ReggeWheeler gauge invariant potentials ${ }^{(i)} f_{\ell 03},{ }^{(i)} f_{\ell 13}$ from (88) and (92). Then with the gauge transformation (94) the resulting perturbations ${ }^{(i)} h_{\ell \mu \nu}$ [cf. (83)-(85)] can be put in asymptotically AdS form.

As was stressed above the special case $\ell=1$ needs a special treatment.

\section{A. the $\boldsymbol{\ell}=1$ case for axial perturbations}

In the $\ell=1$ case the nontrivial equations are $0=$ ${ }^{(i)} E_{113}={ }^{(i)} E_{103}$ and either ${ }^{(i)} f_{103}$ or ${ }^{(i)} f_{113}$ becomes a gauge degree of freedom [i.e., it can be put to zero with a suitable choice of $\left.{ }^{(i)} \eta_{1}\right]$.

If we decide to put ${ }^{(i)} f_{103}$ to zero, then from ${ }^{(i)} E_{113}=0$ we get

$$
\partial_{t}{ }^{(i)} f_{113}={ }^{(i)} f_{1}^{\mathcal{A} \text { res }}+2 A \int{ }^{t}{ }^{(i)} S_{113} d t^{\prime}
$$

where ${ }^{(i)} f_{1}^{\mathcal{A} \text { res }} \equiv{ }^{(i)} f_{1}^{\mathcal{A} \text { res }}(r)$ is set from ${ }^{(i)} E_{103}=0$ :

$$
{ }^{(i)} f_{1}^{\mathcal{A} \text { res }}=\frac{2}{r^{2}} \int_{0}^{r} \frac{r^{\prime 2}}{A}{ }^{(i)} \bar{S}_{103} d r^{\prime}
$$

where ${ }^{(i)} \bar{S}_{103}$ is the time independent part of ${ }^{(i)} S_{103}$ and we used (87).

If we decide to put ${ }^{(i)} f_{113}$ to zero then from ${ }^{(i)} E_{113}=0$ we get

${ }^{(i)} f_{103}={ }^{(i)} f_{1}^{\mathcal{A} \text { res }}-2 r^{2} \int_{\infty}^{r} \frac{A}{r^{\prime 2}}\left(\int{ }^{t}{ }^{(i)} S_{113} d t^{\prime}\right) d r^{\prime}$,

where ${ }^{(i)} f_{1}^{\mathcal{A} \text { res }} \equiv{ }^{(i)} f_{1}^{\mathcal{A} \text { res }}(r)$ can be easily obtained from ${ }^{(i)} E_{103}=0$ :

$$
\partial_{r}\left[\frac{1}{r^{2}} \partial_{r}\left(r^{(i)} f_{1}^{\mathcal{A} \text { res }}\right)\right]=-\frac{2}{r A}{ }^{(i)} \bar{S}_{103},
$$

where ${ }^{(i)} \bar{S}_{103}$ is the time independent part of ${ }^{(i)} S_{103}$ and we used (87).

At linear order the $\ell=1$ part of the perturbation reduces to a pure gauge, as expected [as ${ }^{(1)} S_{\mu \nu} \equiv 0$ ]. 


\section{APPLICATION: PERTURBATIVE CONSTRUCTION OF TIME-PERIODIC SOLUTIONS, PRELIMINARIES}

After discussing our approach to (nonlinear) perturbations of AdS in previous sections, we apply it on some examples. In this section, we present some preliminary results on the perturbative construction of globally regular, time-periodic, aAdS solutions of Einstein equations. Although for more systematic studies of TP solutions it is necessary to go beyond the third order in perturbation expansion (that we postpone for future studies) already the results from the third order can provide some intuitions about TP solutions.

We start with gathering some spectral properties of gravitational perturbations of AdS. Separating the time dependence in the homogeneous wave equation (38) $\tilde{\square}_{\ell} \Phi=0$ in the form $\Phi(t, r)=e(r) \cos (\omega t) \quad$ we get

$$
A\left[-\frac{d}{d r}\left(A \frac{d}{d r} e\right)+\frac{\ell(\ell+1)}{r^{2}} e\right]=\omega^{2} e .
$$

The frequencies $\omega$ are quantized by two boundary conditions: regularity at $r=0$ and the required asymptotic behavior at infinity, (59) and (93) for polar and axial modes, respectively, and the spectra of linear perturbations of AdS (AdS eigenfrequencies) read

$\mathfrak{l} \omega_{\ell, j}^{\mathcal{P}}=1+\ell+2 j \quad$ and $\quad \mathfrak{l} \omega_{\ell, j}^{\mathcal{A}}=2+\ell+2 j$,

where nonnegative integers $j$ are nodal numbers of the corresponding eigenfunctions (AdS eigenmodes)

$$
\begin{aligned}
& e_{\ell, j}^{\mathcal{P}}(r)=\mathcal{N}_{\ell, j}^{\mathcal{P}} \frac{\mathfrak{l} r^{\ell+1}}{\left(\mathfrak{l}^{2}+r^{2}\right)^{\frac{\ell+1}{2}}}{ }_{2} F_{1}\left(-j, 1+\ell+j ; 1 / 2 ; \mathfrak{l}^{2} /\left(\mathfrak{l}^{2}+r^{2}\right)\right), \\
& e_{\ell, j}^{\mathcal{A}}(r)=\mathcal{N}_{\ell, j}^{\mathcal{A}} \frac{\mathfrak{l} r^{\ell+1}}{\left(\mathfrak{l}^{2}+r^{2}\right)^{\frac{\ell+2}{2}}}{ }_{2} F_{1}\left(-j, 2+\ell+j ; 3 / 2 ; \mathfrak{l}^{2} /\left(\mathfrak{l}^{2}+r^{2}\right)\right) .
\end{aligned}
$$

Thus, all AdS eigenfrequencies are real and AdS is linearly stable. The AdS eigenmodes with a given $\ell$ form a complete orthogonal set with respect to the scalar product

$$
(u, v) \equiv \int_{0}^{\infty} \frac{u(r) v(r)}{1+r^{2} / \mathfrak{l}^{2}} d r
$$

namely,

$$
\begin{aligned}
& \left(e_{\ell, j}^{\mathcal{P}}, e_{\ell, k}^{\mathcal{P}}\right)=\frac{\mathfrak{l}^{3} \pi j ! \Gamma(\ell+j+3 / 2)}{2(1+\ell+2 j) \Gamma(j+1 / 2) \Gamma(1+\ell+j)}\left(\mathcal{N}_{\ell, j}^{\mathcal{P}}\right)^{2} \delta_{j k}, \\
& \left(e_{\ell, j}^{\mathcal{A}}, e_{\ell, k}^{\mathcal{A}}\right)=\frac{\mathfrak{l} \pi j ! \Gamma(\ell+j+3 / 2)}{8(2+\ell+2 j) \Gamma(j+3 / 2) \Gamma(2+\ell+j)}\left(\mathcal{N}_{\ell, j}^{\mathcal{A}}\right)^{2} \delta_{j k} .
\end{aligned}
$$

To ease the comparison between our results and those of [13] we take $\mathcal{N}_{\ell, j}^{\mathcal{P}}=\mathcal{N}_{\ell, j}^{\mathcal{A}}=1$ in the following. At any instant of time the master scalar variables ${ }^{(i)} \Phi_{\ell}^{\mathcal{P}}$ and ${ }^{(i)} \Phi_{\ell}^{\mathcal{A}}$ can be expanded in the bases of polar and axial eigenmodes

$$
{ }^{(i)} \Phi_{\ell}^{\mathcal{P} \mid \mathcal{A}}(t, r)=\sum_{j}{ }^{(i)} c_{\ell}^{\mathcal{P} \mid \mathcal{A}}(t) e_{\ell, j}^{\mathcal{P} \mid \mathcal{A}}(r) .
$$

Similarly the inhomogeneous equations (44) and (90) can be projected on the eigenmodes leading to the forced harmonic oscillator equations for Fourier-like coefficients ${ }^{(i)} c_{\ell}^{\mathcal{P} \mid \mathcal{A}}$ :

$$
{ }^{(i)} \ddot{c}_{\ell j}^{\mathcal{P} \mid \mathcal{A}}+\left(\omega_{\ell, j}^{\mathcal{P} \mid \mathcal{A}}\right)^{2}(i) c_{\ell j}^{\mathcal{P} \mid \mathcal{A}}=\left(e_{\ell, j}^{\mathcal{P} \mid \mathcal{A}}, A^{(i)} \tilde{S}_{\ell}^{\mathcal{P} \mid \mathcal{A}}\right)
$$

Since the general solution to the forced harmonic oscillator equation

$$
\ddot{c}(t)+\omega_{0}^{2} c(t)=a \cos (\omega t)
$$


reads

$$
c(t)=\frac{\dot{c}(0)}{\omega_{0}} \sin \left(\omega_{0} t\right)+c(0) \cos \left(\omega_{0} t\right)+ \begin{cases}\frac{a\left(\cos (\omega t)-\cos \left(\omega_{0} t\right)\right)}{\omega_{0}^{2}-\omega^{2}}, & \omega_{0} \neq \omega, \\ \frac{a}{2 \omega_{0}} t \sin \left(\omega_{0} t\right), & \omega_{0}=\omega,\end{cases}
$$

if the projection $\left(e_{\ell, j}^{\mathcal{P} \mid \mathcal{A}}, A^{(i)} \tilde{S}_{\ell}^{\mathcal{P} \mid \mathcal{A}}\right)$ is resonant, i.e., it contains harmonic time dependence $\cos \left(\omega_{\ell, j}^{\mathcal{P} \mid \mathcal{A}} t\right)$ or $\sin \left(\omega_{\ell, j}^{\mathcal{P} \mid \mathcal{A}} t\right)$ then a secular term of the form $t \sin \left(\omega_{\ell, j}^{\mathcal{P} \mid \mathcal{A}} t\right)$ or $t \cos \left(\omega_{\ell, j}^{\mathcal{P} \mid \mathcal{A}} t\right)$ appears in ${ }^{(i)} c_{\ell}^{\mathcal{P} \mid \mathcal{A}}$ and the naive perturbation expansion breaks down. Resumming of all such possible secular terms gives rise to the resonant system (cf. $[7,8]$ in the case of a massless scalar field at spherical symmetry). It may be also possible for some particular first order solution ${ }^{11} \Phi_{\ell}^{\mathcal{P} \mid \mathcal{A}}(t, r)$ to be dressed at higher orders by a suitable choice of frequency corrections and free integration constants [cf. (109)] in such a way, to remove all resonant terms at higher orders and then such ${ }^{(1)} \Phi_{\ell}^{\mathcal{P} \mid \mathcal{A}}(t, r)$ (suitably dressed at higher orders) give rise to TP solutions. For the Einstein-AdS-self-gravitating massless scalar field system, any linear eigenmode can be extended to form the TP solution [2] due to the absence of the so-called $(+++)$ resonances (see [8] for a rigorous theorem). In a recent work [13], Dias and Santos contrasted the gravitational sector of perturbations with the scalar one by noticing that only in special cases (listed in Sec. VI in [13]) linear eigenmodes do admit a nonlinear extension to a regular time-periodic solution. The reason is that at the third order of the perturbation expansion, around most eigenmodes there appear resonant terms that, in contrast to spherically symmetric scalar perturbations studied in [1,2], cannot be removed by a frequency correction. However, this is a purely technical obstruction in constructing TP solutions, due to the degeneracy of the spectrum and, when this degeneracy is properly taken into account, one can construct perturbatively a TP solution bifurcating from each linear eigenfrequency as was pointed out in [14]. Here we complete [14] with some more examples of TP solutions bifurcating from degenerated eigenfrequencies. We aim at constructing a TP solution in the form

$$
\begin{aligned}
\Phi(t, r, \theta)= & { }^{(1)} \Phi(t, r, \theta) \epsilon+{ }^{(2)} \Phi(t, r, \theta) \epsilon^{2}+{ }^{(3)} \Phi(t, r, \theta) \epsilon^{3} \\
& +\mathcal{O}\left(\epsilon^{4}\right)
\end{aligned}
$$

taking for the seed ${ }^{(1)} \Phi$ a linear combination of all polar (axial) modes corresponding to a given eigenfrequency.

a. Polar modes with $\mathfrak{l} \omega^{\mathcal{P}}=5$ as the seed. If we start with

$$
\begin{aligned}
& { }^{(i)} \Phi(t, r, \theta)=\left(\eta e_{2,1}^{\mathcal{P}}(r) P_{2}(\cos \theta)+(1-\eta) e_{4,0}^{\mathcal{P}}(r)\right. \\
& \left.\times P_{4}(\cos \theta)\right) \cos \left(\left(5+{ }^{(2)} \omega \epsilon^{2}\right) t / \mathfrak{l}\right)
\end{aligned}
$$

at linear order [14], then at the third order we get two resonant terms (for the modes $e_{2,1}^{\mathcal{P}}$ and $e_{4,0}^{\mathcal{P}}$ ) that can be removed by a suitable choice of the frequency correction ${ }^{(2)} \omega$ and the mixing parameter $\eta$ in (111). More precisely, the resonant terms will be absent if ${ }^{(2)} \omega$ and $\eta$ satisfy the following system of equations:

$$
\begin{gathered}
-651980329 \eta^{3}+673396185 \eta^{2}-358711575 \eta+22494375=49201152 \eta^{(2)} \omega, \\
16847182891 \eta^{3}-38330631185 \eta^{2}+31825994625 \eta-10200766875=4182097920(1-\eta)^{(2)} \omega .
\end{gathered}
$$

This system has two real solutions: $\left(\eta,{ }^{(2)} \omega\right) \approx(0.1143,-1.900)$ and $\left(\eta,{ }^{(2)} \omega\right) \approx(1.007,-6.487)$; thus, we expect two oneparameter $(\epsilon)$ families of TP solutions to bifurcate from the double eigenfrequency $\mathfrak{l} \omega^{\mathcal{P}}=5$. Note that setting $\eta=1$ in (112), we get ${ }^{(2)} \omega=-34397 / 5376$, while setting $\eta=0$ in (113), we get ${ }^{(2)} \omega=-52311625 / 21446656$, in agreement with the values given in the Table 1 in [13].

b. Polar modes with $\mathfrak{l} \omega^{\mathcal{P}}=6$ as the seed. If we start with

$$
{ }^{(1)} \Phi(t, r, \theta)=\left(\eta e_{3,1}^{\mathcal{P}}(r) P_{3}(\cos \theta)+(1-\eta) e_{5,0}^{\mathcal{P}}(r) P_{5}(\cos \theta)\right) \cos \left(\left(6+{ }^{(2)} \omega \epsilon^{2}\right) t / \mathfrak{l}\right)
$$

at linear order, then at the third order the two potential resonances (for the modes $e_{3,1}^{\mathcal{P}}$ and $e_{5,0}^{\mathcal{P}}$ ) are removed iff the following system holds:

$$
\frac{405\left(11274706467 \eta^{3}-9177443205 \eta^{2}+4622768829 \eta-285923771\right)}{48432676864}=-\frac{315}{128} \eta^{(2)} \omega,
$$




$$
\frac{405\left(1192723542 \eta^{3}-2267107311 \eta^{2}+1606711764 \eta-494724335\right)}{30820794368}=\frac{693}{512}(1-\eta)^{(2)} \omega .
$$

This system has two real solutions: $\left(\eta,{ }^{(2)} \omega\right) \approx(0.1077,-3.770)$ and $\left(\eta,{ }^{(2)} \omega\right) \approx(1.022,-22.92)$, thus we expect two oneparameter families of TP solutions to bifurcate from the double eigenfrequency $\mathfrak{l} \omega^{\mathcal{P}}=6$.

c. Axial modes with $\mathfrak{l} \omega^{\mathcal{A}}=6$ as the seed. If we start with

$$
{ }^{(1)} \Phi(t, r, \theta)=\left(\eta e_{2,1}^{\mathcal{A}}(r) P_{2}(\cos \theta)+(1-\eta) e_{4,0}^{\mathcal{A}}(r) P_{4}(\cos \theta)\right) \cos \left(\left(6+{ }^{(2)} \omega \epsilon^{2}\right) t / \mathfrak{l}\right)
$$

at linear order, then at the third order the two potential resonances (for the modes $e_{2,1}^{\mathcal{A}}$ and $e_{4,0}^{\mathcal{A}}$ ) are removed iff the following system holds:

$$
\begin{gathered}
\frac{239225693 \eta^{3}-398050275 \eta^{2}+217126035 \eta-14644125}{9446621184}=-\frac{35}{384} \eta^{(2)} \omega, \\
\frac{-4829869093 \eta^{3}+13136236344 \eta^{2}-12398007825 \eta+4082879250}{267654266880}=-\frac{63}{512}(1-\eta)^{(2)} \omega .
\end{gathered}
$$

This system has two real solutions: $\left(\eta,{ }^{(2)} \omega\right) \approx(0.1794,-0.08338)$ and $\left(\eta,{ }^{(2)} \omega\right) \approx(1.008,-0.05162)$; thus, we expect two one-parameter families of TP solutions to bifurcate from the double eigenfrequency $\mathfrak{l} \omega^{\mathcal{A}}=6$. Note that setting $\eta=1$ in (118) we get ${ }^{(2)} \omega=-19081 / 376320$, in agreement with the value given in the second line in Table 2 in [13].

d. Axial modes with $\mathfrak{l} \omega^{\mathcal{A}}=7$ as the seed. If we start with

$$
{ }^{(1)} \Phi(t, r, \theta)=\left(\eta e_{3,1}^{\mathcal{A}}(r) P_{3}(\cos \theta)+(1-\eta) e_{5,0}^{\mathcal{A}}(r) P_{5}(\cos \theta)\right) \cos \left(\left(7+{ }^{(2)} \omega \epsilon^{2}\right) t / \mathfrak{l}\right)
$$

at linear order, then at the third order the two potential resonances (for the modes $e_{3,1}^{\mathcal{A}}$ and $e_{5,0}^{\mathcal{A}}$ ) are removed iff the following system holds:

$$
\begin{aligned}
& \frac{1978248043512 \eta^{3}-2997546380577 \eta^{2}+1613025400770 \eta-106858809617}{56956827992064}=-\frac{21}{256} \eta^{(2)} \omega, \\
& \frac{3608791248537 \eta^{3}-9113490099567 \eta^{2}+8191761299127 \eta-2669524922785}{144981016707072}=\frac{231}{2048}(1-\eta)^{(2)} \omega .
\end{aligned}
$$

This system has two real solutions: $\left(\eta,{ }^{(2)} \omega\right) \approx$ $(0.1670,-0.1130)$ and $\left(\eta,{ }^{(2)} \omega\right) \approx(1.018,-0.1085)$; thus, we expect two one-parameter families of TP solutions to bifurcate from the double eigenfrequency $\mathfrak{l} \omega^{\mathcal{A}}=7$.

e. Some general remarks. In general, for the eigenfrequency with geometric multiplicity $k$ we need to take a linear combination of $k$ corresponding eigenmodes as the seed, and to remove the resonances at the third order we have to fulfill the system of $k$ equations that are cubic in the mixing parameters $\eta_{1}, . ., \eta_{k-1}$ and linear in ${ }^{(2)} \omega \eta_{i}$ terms (with $1 \leq i \leq k-1$ ). Each real root of this system gives rise to a TP solution bifurcating from the given eigenfrequency [the number of all (complex) roots is expected to grow exponentially with $k$, as after eliminating ${ }^{(2)} \omega$, we are left with the system of $k-1$ equations that are quartic in $\left.\eta_{1}, \ldots, \eta_{k-1}\right]$. Interestingly, in all cases that we have studied so far $\left(\mathfrak{l} \omega^{\mathcal{P}}=3,4,5,6,7,8\right.$ and $\mathfrak{l} \omega^{\mathcal{A}}=4,5,6,7,8$ at axial symmetry), the number of bifurcating one-parameter families of time-periodic solutions is equal to the multiplicity of the eigenfrequency. This intriguing coincidence deserves further studies. Finally, we remark that in a parallel work Maliborski constructed axially symmetric time-periodic solutions for the cubic wave equation on the fixed AdS background [29], and in that model there is no such coincidence.

\section{CONCLUSIONS}

We have presented the formalism for nonlinear gravitational perturbations of AdS spacetime and applied it to provide the evidence for the existence and properties of globally regular, asymptotically AdS, time-periodic solutions of vacuum Einstein equations. These time-periodic solutions bifurcate from the linear eigenfrequencies of $\mathrm{AdS}$, and the number of (one-parameter families of) such solutions bifurcating from a given eigenfrequency equals the multiplicity of this eigenfrequency. This intriguing 
coincidence deserves further study. Already the results obtained at third order should provide the initial guess for the numerical method of [13], which is good enough for this method to converge and to provide an independent cross-check of our results. The presented formalism should form the solid base for the future construction of the resonant system for vacuum Einstein-AdS equations, and the more systematic study of time-periodic solutions.

Although we were mainly motivated by the gravitational perturbations of AdS, all the formulas in the paper that are not related to the asymptotic structure of spacetime are general enough to encompass gravitational perturbations of the other two maximally symmetric vacuum solutions of Einstein equations, i.e., Minkowski and de Sitter spacetimes, and with some modifications also spherically symmetric vacuum solutions and as such will hopefully find application in the broad subject of perturbations of such spacetimes. It would be also very interesting to introduce matter in this context into the presented formalism.

\section{ACKNOWLEDGMENTS}

This research was supported in part by the Polish National Science Centre Grant No. DEC-2012/06/A/ST2/ 00397. The author is indebted to Piotr Bizon and Maciej Maliborski for many valuable discussions and to Piotr Bizon for many valuable remarks on the early version of the manuscript. The author acknowledges the six month scientific associate contract at the CERN TH department that allowed him the detailed studies of gravitational perturbations of AdS, and is particularly grateful to Luis Álvarez-Gaumé for providing a propitious atmosphere for the research.

Note added.-A few days after the first arXiv version of this paper was released, there appeared a very interesting parallel work [30] on an AdS gravitational geons. In particular, in [30] the families of excited geons, bifurcating from degenerated linear frequencies of AdS, were numerically constructed.
[1] P. Bizoń, A. Rostworowski, Weakly Turbulent Instability of Anti-de Sitter Spacetime, Phys. Rev. Lett. 107, 031102 (2011).

[2] M. Maliborski and A. Rostworowski, Time-Periodic Solutions in an Einstein AdS-Massless-Scalar-Field System, Phys. Rev. Lett. 111, 051102 (2013).

[3] M. Maliborski and A. Rostworowski, A comment on Boson stars in AdS, arXiv:1307.2875.

[4] A. Buchel, S. L. Liebling, and L. Lehner, Boson stars in AdS spacetime, Phys. Rev. D 87, 123006 (2013).

[5] O. J. C. Dias, G. T. Horowitz, D. Marolf, and J. E. Santos, On the nonlinear stability of asymptotically anti-de Sitter solutions, Classical Quantum Gravity 29, 235019 (2012).

[6] M. Maliborski and A. Rostworowski, What drives AdS spacetime unstable?, Phys. Rev. D 89, 124006 (2014).

[7] V. Balasubramanian, A. Buchel, S. R. Green, L. Lehner, and S. L. Liebling, Holographic Thermalization, Stability of AdS, and the Fermi-Pasta-Ulam-Tsingou Paradox, Phys. Rev. Lett. 113, 071601 (2014).

[8] B. Craps, O. Evnin, and J. Vanhoof, Renormalization group, secular term resummation and AdS (in)stability, J. High Energy Phys. 10 (2014) 048.

[9] B. Craps, O. Evnin, and J. Vanhoof, Renormalization, averaging, conservation laws and AdS (in)stability, J. High Energy Phys. 01 (2015) 108.

[10] P. Bizoń, M. Maliborski, and A. Rostworowski, Resonant Dynamics and the Instability of Anti-de Sitter Spacetime, Phys. Rev. Lett. 115, 081103 (2015).

[11] G. T. Horowitz and J.E. Santos, Geons and the instability of anti-de Sitter spacetime, Surv. Differ. Geom. 20, 321 (2015).
[12] O. J. C. Dias, G. T. Horowitz, and J. E. Santos, Gravitational turbulent instability of Anti-de Sitter space, Classical Quantum Gravity 29, 194002 (2012).

[13] O. J. C. Dias and J. E. Santos, AdS nonlinear instability: Moving beyond spherical symmetry, Classical Quantum Gravity 33, 23LT01 (2016).

[14] A. Rostworowski, Comment on AdS nonlinear instability: Moving beyond spherical symmetry, Classical Quantum Gravity 34128001 (2017).

[15] O. Evnin and R. Nivesvivat, AdS perturbations, isometries, selection rules and the Higgs oscillator, J. High Energy Phys. 01 (2016) 151.

[16] T. Regge and J. A. Wheeler, Stability of a Schwarzschild singularity, Phys. Rev. 108, 1063 (1957).

[17] H.-P. Nollert, Quasinormal modes: The characteristic sound of black holes and neutron stars, Classical Quantum Gravity 16, R159 (1999).

[18] S. Mukohyama, Gauge-invariant gravitational perturbations of maximally symmetric spacetimes, Phys. Rev. D 62 , 084015 (2000).

[19] F. J. Zerilli, Effective Potential for Even-Parity ReggeWheeler Gravitational Perturbation Equations, Phys. Rev. Lett. 24, 737 (1970).

[20] M. Bruni, S. Matarrese, S. Mollerach, and S. Sonego, Perturbations of spacetime: Gauge transformations and gauge invariance at second order and beyond, Classical Quantum Gravity 14, 2585 (1997).

[21] A. Garat and R. H. Price, Gauge invariant formalism for second order perturbations of Schwarzschild spacetimes, Phys. Rev. D 61, 044006 (2000). 
[22] We recall that here, and in the following, that $\Delta_{L}{ }^{(i)} h_{\ell \mu \nu}$ refers to the expansion of the tensor $\Delta_{L}{ }^{(i)} h_{\mu \nu}^{(i)}$ according to (11)-(14) and not the action of the $\Delta_{L}$ operator on ${ }^{(i)} h_{\ell \mu \nu}(t, r)$.

[23] H. Kodama and A. Ishibashi, A master equation for gravitational perturbations of maximally symmetric black holes in higher dimensions, Prog. Theor. Phys. 110, 701 (2003).

[24] Strictly speaking

$$
\tilde{\square}_{\ell} \Phi(t, r)=r\left(-\bar{\square}+V_{\ell}\right) \frac{\Phi(t, r)}{r},
$$

where $\bar{\square}$ is the wave operator for the metric (10) and the potential $V_{\ell}$ reads

$$
V_{\ell}=\frac{\ell(\ell+1)}{r^{2}}-\frac{A^{\prime}}{r} .
$$

[25] D. Brizuela, J. M. Martín-García, and M. Tiglio, Complete gauge-invariant formalism for arbitrary second-order perturbations of a Schwarzschild black hole, Phys. Rev. D 80, 024021 (2009).

[26] V. Moncrief, Gravitational perturbations of spherically symmetric systems. I. The exterior problem, Ann. Phys. (N.Y.) 88, 323 (1974).

[27] M. Henneaux and C. Teitelboim, Asymptotically anti-de Sitter spaces, Commun. Math. Phys. 98, 391 (1985).

[28] H. Bantilan, F. Pretorius, and S. Gubser, Simulation of asymptotically $\mathrm{AdS}_{5}$ spacetimes with a generalized harmonic evolution scheme, Phys. Rev. D 85, 084038 (2012).

[29] M. Maliborski, Cubic wave equation on AdS with axial symmetry a toy model of geons (to be published).

[30] G. Martinon, G. Fodor, P. Grandclément, and P. Forgács, Gravitational geons in asymptotically anti-de Sitter spacetimes, Classical Quantum Gravity 34, 125012 (2017). 